\title{
Role of Ultrasonography in the Era of 3 Tesla MRCP: A Comparative Study in Obstructive Jaundice.
}

\author{
Vikram Patil ${ }^{1}$, Sudha Kiran Das ${ }^{1}$, Devkant ${ }^{1}$, Rudresh Hiremath ${ }^{1}$, Sachin Shetty ${ }^{1}$, Vinay Raj ${ }^{1}$ \\ 'JSS Medical College, JSS AHER, Mysuru.
}

\section{Abstract}

Background: Biliary obstruction is caused by various diseases. In a suspected case of biliary obstruction with clinical and laboratory findings suggestive of obstructive jaundice, the main aim of radiologist is to confirm the presence of obstruction identify its location, extent and probable cause. 3D MRCP virtually demonstrates anatomy of the biliary and pancreatic ducts in 3 Tesla MRI system. Ultrasonography still remains the first imaging modality for obstructive jaundice. The aim of the study was to evaluate the diagnostic efficacy of USG vs. MRCP with 3 Tesla MRI in patients of obstructive jaundice. Subjects and Methods: After institutional ethical committee approval, 90 patients confirmed to have features of obstructive jaundice referred for Ultrasonography and MRCP and subsequently underwent ERCP / Surgery were evaluated. It was a Prospective correlational study. Inclusion Criteria were patients with clinical diagnosis of obstructive jaundice referred for Ultrasonography and MRCP and subsequently underwent ERCP / Surgery. Exclusion Criteria were patients non compatible for MRI and other modalities (ERCP), Patients in whom histological diagnosis was not available, Patients unwilling for MRCP / ERCP / Surgery. Results: Accuracy of ultrasound was $75 \%$ and MRCP $98 \%$ in detection and delineation of obstructive biliary pathologies. Sensitivity, specificity and accuracy for choledocholithiasis is $80 \%, 100 \%$ \& $80 \%$ respectively for Ultrasonography and $100 \%$ for MRCP. The Sensitivity and Specificity for Ultrasonography was $71 \%$ and $100 \%$ in detection of biliary obstruction secondary to biliary strictures and for MRCP, the sensitivity and specificity was $100 \%$. Sensitivity and specificity for malignant pathologies in this study was $73 \%$ and $100 \%$ respectively on Ultrasonography, while that on MRCP was $93.3 \%$ and $100 \%$ respectively. Conclusion: Ultrasonography as a screening modality is useful to confirm or exclude biliary dilatation and to choose patients for MRCP examination. MRCP is an important non-invasive imaging investigation in the pre-operative evaluation of patients with obstructive jaundice. Magnetic resonance (MR) cholangiopancreatography, combines the advantages of projectional imaging with those of cross-sectional imaging, is an established diagnostic technique. Technical advances over the past decade, including the refinement of 3.0-T imaging capabilities, have added to the usefulness of this noninvasive method. Though MRCP can be considered as the gold standard for diagnostic non-invasive evaluation of the pancreaticobiliary system in cases of obstructive jaundice, ultrasound will always remain the first choice.

Keywords: Ultrasonography, MRSA, Obstructive Jaundice.

Corresponding Author: Dr. Vikram Patil, JSS Medical College, JSS AHER, Mysuru

Received: February 2019

Accepted: February 2019

\section{Introduction}

Evaluation of a suspected obstructive biliary tract disease is a common radiological problem and traditionally diagnosed by a variety of imaging modalities including Ultrasonography (USG), Computed tomography (CT), Invasive Cholangiography and Magnetic Resonance Cholangio Pancreatography (MRCP). Biliary obstruction is caused by various diseases such as common bile duct stone, post inflammatory or postsurgical strictures, sclerosing cholangitis, Mirizzi's syndrome and malignant pancreaticobiliary neoplasm. ${ }^{[1]}$

In a suspected case of biliary obstruction with clinical and laboratory findings suggestive of obstructive jaundice, the main aim of radiologist is to confirm the presence of obstruction identify its location, extent and probable cause. In suspected biliary obstruction Ultrasonography is used as the initial screening procedure due to its many advantages like its ready availability, its cost effectiveness. ${ }^{[2]}$

MRCP has advantages of complete mapping of the ductal system, non-invasiveness, no requirement of contrast material and its ability to produce highly accurate cholangiographic images, similar in appearance to those obtained by invasive procedures like Endoscopic Retrograde Cholangiopancreatography (ERCP),yet combining the patient safety and comfort associated with ultrasound. ${ }^{[3]}$

3D MRCP virtually demonstrates anatomy of the biliary and pancreatic ducts in 3 Tesla MRI system. Various sequences are used, but mainly all rely on extreme T2 weighting. Thick slab single- shot FSE images and thin multislice HASTE images are both significantly contributory and reduces total time in imaging with 3 Tesla MRI system. The thick slab technique provides an overview of bile duct anatomy in $<5$ minutes and is suitable. Thick slab images are obtained with a long TE $(940 \mathrm{~m} \mathrm{sec})$ to provide complete suppression of background tissue. All 
images are acquired during breath-holding, with orientation determined by initial review of the individual anatomy of the gland direct coronal or oblique images are often most useful. $^{[4]}$

Key to achieving the proper diagnosis with a dilated bile duct is evaluating the zone of transition from dilated to nondilated or non-visualized duct. Special attention should be paid to this zone of transition regardless of imaging modality being used. With a borderline or equivocally increased extra hepatic duct diameter, re-evaluation of duct size following a fatty meal can help differentiate obstructed from non-obstructed ducts.

At the time of first clinical application of MRCP over two decades ago, MRCP was regarded as best, as a novel technique and with a questionable potential for imaging the biliary and pancreatic duct. MRCP is performed by using heavily $\mathrm{T} 2 \mathrm{~W}$ turbo spin echo sequences and fast gradient sequences, in which stationary fluids has resultant high signal intensity. ${ }^{[6]}$

MRCP takes the advantage of high signal intensity of body fluids on heavily T2W MRI. Static or slow moving fluid filled structures such as bile and pancreatic ducts appear hyper intense areas, where as background tissues generate some signal. This inherent difference in signal intensity enables MRCP to be carried out without any contrast agents. ${ }^{[7]}$

Fast spin echo sequences were less susceptible to motion artifacts, slow flow and magnetic susceptibility. The signal to noise ratio and contrast to noise ratio was significantly higher than SSFP sequences. Initially 2D fast spin echo sequences were introduced; subsequently 3D FSE respiratory triggered sequences were introduced permitting thinner slices. $^{[8]}$

Modified FSE sequences were introduced recently. These are RARE (Rapid acquisition with relaxation enhancement) and Half Fourier acquisition single shot turbo spin echo sequences (HASTE) ${ }^{[9]}$ HASTE is Half Fourier acquisition single shot turbo spin echo sequences, multiple thin slices ranging from $2-7 \mathrm{~cm}$ in thickness are obtained in 18-20 seconds breath hold. This technique has advantages like RARE to eliminate respiratory motion artifacts as well as negligible magnetic susceptibility artifacts from the bowel loops and surgical clips. Multislice HASTE sequences should still be acquired if choledocholithiasis is suspected. The diagnostic efficiency of single shot RARE sequences in pancreatic duct and intra hepatic duct disease is still being validated. ${ }^{[10]}$

The 3D MRCP sequences had a similarly high sensitivity and specificity for detection of choledocholithiasis. ${ }^{[1]}$

For evaluation of pancreatic parenchyma Gadolinium enhanced images are acquired. Combination of phased array coil and rapid sequences enables detection of $1 \mathrm{~mm}$ ducts. ${ }^{[12]}$ with phased array coil, abdominal wall motion and the respiratory artifacts are reduced. Disadvantages are the incomplete coverage of the abdomen, the inhomogeneous signal intensity and the expenses of the additional system. The limitation of MRCP is that ascites or fluid collection may obscure ductal anatomy, which however may be partly overcome by use of multi- oblique method. ${ }^{[13]}$

\begin{tabular}{|c|c|c|c|}
\hline \multicolumn{4}{|c|}{ Common causes of biliary obstruction } \\
\hline $\begin{array}{c}\text { Intra hepatic biliary obstruction } \\
\text { obstruction }\end{array}$ & Porta hepatic biliary obstruction & $\begin{array}{c}\text { Supra pancreatic } \\
\text { biliary obstruction }\end{array}$ & $\begin{array}{c}\text { Intra pancreatic } \\
\text { Biliary obstruction }\end{array}$ \\
\hline Primary sclerosing cholangitis & Cholangiocarcinoma & Pancreatic carcinoma & Pancreatic carcinoma \\
\hline Space occupying lesions and liver diseases & Primary sclerosing cholangitis & Metastasis & Pancreatitis \\
\hline & Primary Carcinoma GB & Pancreatitis & Choledocholithiasis \\
\hline & Metastatic diseases & Cholangiocarcinoma & Ampullary Carcinoma \\
\hline & Strictures & Choledocholithiasis & Duodenal Carcinoma \\
\hline & & Strictures & Cholangiocarcinoma \\
\hline
\end{tabular}

\section{Anatomy of biliary tract:}

About 3-4 $\mathrm{cm}$ after leaving the liver, the CHD joins the cystic duct to form CBD. The length of $\mathrm{CBD}$ varies from 5$15 \mathrm{~cm}$, depending on where CHD and cystic ducts join. ${ }^{[14]}$ The cystic duct attaches the gall bladder to the extra hepatic bile duct; its point of insertion into the extra hepatic bile duct marks the division between the common hepatic duct and common bile duct. The cystic duct usually joins the extra hepatic bile duct approximately halfway between the porta hepatis and ampulla of Vater. ${ }^{[15]}$

\section{Variation in Cystic duct insertion:}

Congenital anatomic variants of cystic ducts are common, occurring in $18-23 \%$ of cases. the cystic duct inserts into the middle $1 / 3 \mathrm{rd}$ of the extra hepatic bile duct in $75 \%$ cases and into the distal $1 / 3 \mathrm{rd}$ in $10 \%$ cases. ${ }^{[15]}$

Cystic ducts stones in a low, medially inserting cystic duct that joins the common bile duct at the ampulla may be mistaken from stone in the distal bile duct or may result in bile obstruction or gall stone pancreatitis. Unusually long cystic duct remnant (upto $6 \mathrm{~cm}$ in length) may be associated with inflammatory changes and formation of calculi, resulting in post cholecystectomy syndrome, cause of the persistent and recurrent biliary symptoms in affected patients. $^{[15]}$

Ultrasonography \& MRI appearance of normal cystic duct: Ultrasonography appearance: In most cases, the normal cystic duct is not seen at Ultrasonography. However, with optimal technique, the normal cystic duct can be visualized in upto $50 \%$ cases as an anechoic tubular structure connection gall bladder CBD bilc duct. A cystic duct the runs parallel to the distal extra hepatic bile duct may be confused with a vessel; however differentiation is possible with doppler Ultrasonography. ${ }^{[15]}$

MRCP appearance: The cystic duct is seen at MRCP in virtually all cases. The capacity of MRCP to provide coronal images and to orient the angle of image acquisition along the long axis of cystic duct optimizes duct 


\section{Patil et al; Rale of Ultrasanagraphy in the Era of 3 Jesla MRCP}

visualization. Cystic ducts is routinely seen in MRCP and can be traced to its junction with extra hepatic bile duct in most cases. ${ }^{[15]}$

\section{Anomalies of biliary tree:}

Dilated intrahepatic ducts should be diagnosed when the intrahepatic bile ducts exceeds $>2 \mathrm{~mm}$ or $40 \%$ of the diameter of the adjacent intrahepatic portal vein.16 Aberrant or accessory biliary ducts may predispose patients to inadvertent ductal ligation at laparoscopic cholecystectomy and may complicate surgeries, such as living donor right lobe liver transplantation. ${ }^{[16]}$

\section{Aberrant Biliary Ducts:}

Anatomic variations of the biliary tract occur commonly, normally right and left hepatic ducts converge at the porta hepatis to form the common hepatic duct.

\section{PANCREAS:}

Pancreas is located retroperitoneally in the anterior pararenal space posterior to the stomach and bounded by the c-loop of duodenum on the right side. The parts of pancreas are head, uncinate process, neck, body, and tail. ${ }^{[17]}$

Pancreatic Anomalies: Congenital anomalies and normal variants of the pancreatic duct and the pancreas may not be detected until adulthood and then are often detected as incidental findings in asymptomatic patients. Congenital anomalies and normal variants of pancreas and the pancreatic duct include pancreas divisum, annular pancreas, ectopic pancreatic tissue, variation of pancreatic contour, fatty replacement and fat sparing of pancreas, pancreatic cysts and variation of pancreatic ducts. ${ }^{[18]}$

\section{Pancreatic Divisum:}

The main features of pancreas divisum when using MRCP include the dorsal pancreatic duct in directly continuity with the duct of santorini, which drains into the minor ampulla and ventral duct, which does not communicate with the distal dorsal duct but joins with distal bile duct to enter the major ampulla. ${ }^{[18]}$

\section{Anomalous junction of the biliary ductal system and pancreatic duct:}

Anomalous junction of pancreatico biliary system is usually defined as a union of distal CBD and the pancreatic duct proximal to the duodenum whose length is greater the $1.5 \mathrm{~cm}$. Anomalous junction of the pancreaticobiliary ductal system has been reported in association with cholangiocarcinoma, gall bladder carcinoma, choledocholithiasis and chronic pancreatitis. ${ }^{[19]}$

\section{Pancreatic pathologies:}

* Inflammatory disorders: Acute pancreatitis, Chronic pancreatitis, Groove pancreatitis, Autoimmune pancreatitis

* Neoplastic etiology: Carcinoma of Pancreas, Peri ampullary carcinoma

Normal Ultrasonography \& MR imaging appearances of pancreas and pancreatic duct:

- Ultrasonography appearance: The pancreas is slightly echogenic compared to the liver and has a homogenous appearance. Pancreatic duct appears as an anechoic linear tubular structure in the pancreatic tissue.

- MRI appearance: The pancreas is unique in that it has the highest 'I'l signal intensity of intra-abdominal organs on the fat suppressed sequences higher than or atleast equal to liver in signal intensity. The high signal intensity persists even with fatty infiltration in which case pancreas exhibits a marbled appearance. The main pancreatic duct courses cephalad, takes a 45-90 degree turn in the neck and continues horizontally in the body and in the tail of the gland. The normal diameter is between 2 to $3 \mathrm{~mm}$. It unites with CBD to form hepatopancreatic ampulla. ${ }^{[17]}$

\section{Common bile duct:}

The CBD is nearly always visualized coursing through the porta hepatis at Ultrasonography. The upper limit of its diameter has been a source of controversy for decades relating to the variation among individuals and whether one wishes to correlate diameter with obstruction with high sensitivity and specificity. Generally at ultrasound, 6-7 mm in diameter has come to be the most commonly used upper limit size. ${ }^{[20]}$

There is also some controversy over whether the EHBR increases its diameter in response to cholecystectomy and in aging. A study done by Bachar ct al has revealed an age dependent change in the diameter of the EHBD. The study suggests the upper normal limit of the duct in elderly persons be set at $8.5 \mathrm{~mm} .^{[21]}$

\section{Ultrasonography \& MRI appearance of normal} Common bile duct:

Ultrasonography appearance: Linear tubular structure with anechoic lumen lying anterior to portal vein at porta hepatis. MRCP appearance: On T2 WI it appears hyperintense tubular structure extending caudally within the hepatoduodenal ligament, lying anterior to portal vein and to the right of hepatic artery. It then passes posterior to the first portion of duodenum and the head of pancreas, sometimes embedded in the latter.

Pathologies of Common bile duct: Choledocholithiasis, Choledochal cysts, Caroli's disease, Recurrent Pyogenic Cholangitis, Primary Sclerosing Cholangitis, Cholangiocarcinoma,CBD strictures

\section{GALL BLADDER:}

The gallbladder is a pear-shaped hollow viscus located in the right upper quadrant, lodged on the visceral surface of the liver between segments IV and V, and connected to the hepatic duct through the cystic duct to form the common bile duct. It is usually $7-10 \mathrm{~cm}$ long and $2.5 \mathrm{~cm}$ wide, and the wall measures less than $3 \mathrm{~mm}$ in thickness. ${ }^{[22]}$

\section{Anatomical variants: \\ Anomalous positions and agenesis of the gallbladder is rare. Imaging of multiple gallbladders is challenging, since the duplicated gallbladder(s) are difficult to detect on sonography, oral cholecystography (OCG), and scintigraphy. ${ }^{[23]}$}

Ultrasonography and MRI appearance of normal Gall 


\section{bladder:}

Ultrasonography appearance: A normal Gallbladder should be thin walled $(<3 \mathrm{~mm})$ and anechoic. It is a pear shaped saccular organ lying in the inferior margin of liver between right and left lobes. ${ }^{[19]}$

MRI appearance: On T2-weighted images, the gallbladder wall has low signal intensity and stands out against the bright visceral fat. The wall adjacent to the liver cannot be identified as a separate structure. The insertion of the cystic duct into the hepatic duct can be demonstrated with routine T2-weighted imaging and MR cholangiography. Normal bile appears uniformly bright with T2-weighted sequences. On T1-weighted images, bile varies greatly in signal intensity depending on its concentration. During fasting, bile undergoes a process of concentration. Water is reabsorbed and the concentration of cholesterol and bile salts increases, leading to a shortened $\mathrm{T} 1$ relaxation time and, consequently, to bright bile on T1-weighted images. A layering effect is sometimes observed, with concentrated and denser bile in the dependent position. Gallbladder sludge may have similar signal intensity characteristics, namely, iso- to mild hyperintensity on T2-weighted images and hyperintensity on T1-weighted images. ${ }^{[22]}$

\section{Aims \& Objective}

- To evaluate the diagnostic efficacy of USG vs. MRCP with 3 tesla MRI in patients of obstructive jaundice.

- To correlate ultrasonography and MRCP findings with ERCP/Surgical outcome and histopathology.

\section{Subjects and Methods}

The Study was conducted with the approval of the institutional ethical committee on 90 patients confirmed to have features of obstructive jaundice referred for Ultrasonography and MRCP to Department of RadioDiagnosis and subsequently underwent ERCP / Surgery followed by histopathological after informed consent Type of study: Prospective and correlational study.

\section{Inclusion Criteria:}

- Patients with clinical diagnosis of obstructive jaundice who were referred for Ultrasonography and MRCP to Department of Radio-Diagnosis and subsequently underwent ERCP / Surgery.

\section{Exclusion Criteria:}

- Patients non compatible for MRI and other modalities (ERCP).

- Patients in whom histological diagnosis was not available / inoperable.

- Patients unwilling for MRCP / ERCP / Surgery.

Imaging protocols:

Patients with features of surgical obstructive jaundice who are referred to the Radio-Diagnosis department are subjected to Ultrasonography and MRI according to the following protocols:

\section{Protocol for ultrasonography:}

Abdomen of all patients are examined after 6-10 hours of fasting in B-mode with curvilinear probe with wave frequency of $5 \mathrm{MHz}$. All Ultrasonography's were done either in iU22 or HD11XE Philips machines. Linear probe with wave frequency of $10 \mathrm{MHz}$ was used in thin patients.

\section{Protocol for MRCP:}

Equipment : 3 Tesla PHILIPS INGENIA, Wholebody MR scanner

Patients are kept nil by mouth fasted for 4-6 hours prior to the study in order to reduce fluid secretions within the stomach and duodenum, reduce bowel peristalsis and promote gallbladder distension. A negative oral contrast agent was given to reduce the signal intensity of overlapping fluid within the stomach and duodenum. Ear plugs are given to patient to avoid any hearing damage and the following sequences of images are taken coronal T2W SPAIR, SAG T2W TSE, TRANS T2W SPAIR, TRANS T1 TFE, SMRCP 3D,SSFSE MRCP.

\section{Statistics}

\section{Data Analysis and Interpretation:}

Data was entered into Microsoft Excel (Windows 7; Version 2007) and analyses were done using the Statistical Package for Social Sciences (SPSS) for Windows software (version 22.0; SPSS Inc, Chicago). Descriptive statistics such as mean and standard deviation (SD) for continuous variables, frequencies and percentages were calculated for categorical Variables were determined. Association between Ultrasound and MRCP Variables were analysed using chi-square test. Pearson Correlation coefficient was used to show correlation between Obstructive jaundice and other quantitative variables. Level of significance was set at 0.05 .

\section{Results}

The introduction of MRCP readily permits the study of anatomy and pathology of the biliary tree including pancreatic duct very easily which is utmost important for diagnosing the cause of obstructive jaundice.

A total of 90 patients of obstructive jaundice who underwent both Ultrasonography and MRCP, ERCP examinations / surgery, were chosen for the study. Majority of pathologies observed were benign (83.3\%). Most common benign disorder observed was choledocholithiasis $(55.6 \%)$ followed by benign strictures $(23.3 \%)$. This also included two cases of Mirizzi syndrome and two cases of choledochal cysts.

Table 1: Causes of Obstructive Jaundice in studied Population.

\begin{tabular}{|c|c|c|}
\hline Pathology & No. of Cases & Percentage (\%) \\
\hline Benign strictures & 21 & 23.3 \\
\hline Choledocholithiasis & 50 & 55.6 \\
\hline Choledochal cyst & 2 & 2.2 \\
\hline Mirizzi Syndrome & 2 & 2.2 \\
\hline Cholangiocarcinoma & 5 & 5.6 \\
\hline Periampullary carcinoma & 5 & 5.6 \\
\hline Carcinoma Gall bladder & 3 & 3.3 \\
\hline Carcinoma pancreas & 2 & 2.2 \\
\hline
\end{tabular}


Overall accuracy of ultrasound was $75 \%$ and MRCP $98 \%$ in detection and delineation of obstructive biliary pathologies. Sensitivity, specificity and accuracy for choledocholithiasis is $80 \%, 100 \% \& 80 \%$ respectively for Ultrasonography and 100\% for MRCP. The Sensitivity and Specificity for Ultrasonography was $71 \%$ and $100 \%$ in detection of biliary obstruction secondary to biliary strictures and for MRCP, the sensitivity and specificity was $100 \%$. Sensitivity and specificity for malignant pathologies in this study was $73 \%$ and $100 \%$ respectively on Ultrasonography, while that on MRCP was $93.3 \%$ and $100 \%$ respectively.

Table 2: Detection of Obstructive pathology: Modality wise

\begin{tabular}{|c|c|c|c|c|c|c|}
\hline Pathology & $\begin{array}{c}\text { USG } \\
(\mathbf{9 0})\end{array}$ & $\begin{array}{c}\text { MRCP } \\
\mathbf{( 9 0 )}\end{array}$ & $\mathbf{\%}$ & $\begin{array}{c}\text { Surgery } \\
\mathbf{( 9 0 )}\end{array}$ & $\mathbf{\%}$ \\
\hline Choledocholithiasis & 40 & 44.4 & 50 & 55.6 & 50 & 55.6 \\
\hline Benign strictures & 15 & 16.6 & 22 & 24.4 & 21 & 23.3 \\
\hline Cholangiocarcinoma & 3 & 3.3 & 5 & 5.6 & 5 & 5.6 \\
\hline $\begin{array}{c}\text { Periampullary } \\
\text { carcinoma }\end{array}$ & 3 & 3.3 & 4 & 4.5 & 5 & 5.6 \\
\hline $\begin{array}{c}\text { Carcinoma Gall } \\
\text { bladder }\end{array}$ & 3 & 3.3 & 3 & 3.3 & 3 & 3.3 \\
\hline $\begin{array}{c}\text { Carcinoma pancreas } \\
\text { Choledochal cyst }\end{array}$ & 2 & 2.2 & 2 & 2.2 & 2 & 2.2 \\
\hline Mirizzi syndrome & 3 & 2.2 & 2 & 2.2 & 2 & 2.2 \\
\hline
\end{tabular}

\section{Discussion}

While evaluating a case of obstructive jaundice, the radiologist aims at confirming the presence of obstruction, identify its location, extent and probable cause. Ultrasonography has traditionally been used as the initial screening procedure because of its many advantages, which include its ready availability, cost effectiveness, no requirement of contrast material and lack of ionizing radiation. However, although it is well suited to visualize the Common Hepatic Duct (CHD) and proximal CBD, one of its major limitations is assessment of the distal CBD and pancreas due to poor acoustic window secondary to overlying bowel gas \& obesity.

In this study, 90 patients with clinical diagnosis of obstructive jaundice were evaluated with ultrasound, MR and ERCP/Surgery. Out of the 90 Patients evaluated, $40(44.4 \%)$ patients were female and $50(55.6 \%)$ patients were male. The mean age of study population is 54.6 (range $1-83 y r s$ ), the youngest was a year old girl with Choledochal cyst and the oldest was a 83year old female benign biliary stricture. Ultrasonography was done prior to MRCP, followed by Surgery/ ERCP/FNAC/Biopsy.

Of the 90 patients evaluated, 15 patients had malignancy as causes of biliary obstruction, while in the rest 75 patients, the etiology was benign. The most common etiopathogenesis of obstruction noted in this study was choledocholithiasis $(50 / 75)$ followed by benign biliary stricture (21/75), Choledochal cyst (2/75) and Mirizzi's $(2 / 75)$. Of the 15 patients with malignant causes for obstruction, commonest was periampullary carcinoma $(5 / 15)$, cholangiocarcinoma $(5 / 15)$, Carcinoma GB (3/15) \& carcinoma head of pancreas $(2 / 15)$ all of which were confirmed on Surgery/ ERCP/Biopsy/FNAC.

Of the 50 cases Choledocholithiasis, Sensitivity and Specificity of sonographic detection was $79 \%$ \& $100 \%$ respectively and for MRCP sensitivity \& specificity was $100 \%$. Observed values in this study is in concordance with the previous studies, of noteworthy is one metanalysis by Abboud PA et al, ${ }^{[24]}$ the sonographic sensitivity varied from $55-99 \%$, this variation was attributed to technical limitations (poor acoustic window, obesity, tissue interfaces) and expertise.
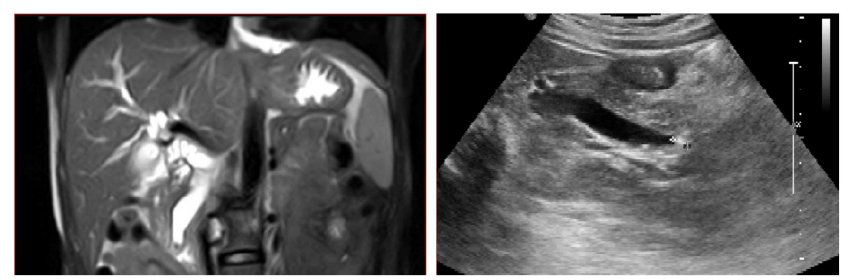

Figure 1: USG Showing dilated CBD with distal CBD calculus. T2 weighted fat saturated image Coronal section shows dilated CBD with a signal void distal CBD calculus.

Of 22 patients with CBD stricture, accurate sonographic detection was done only in 15 patients, whereas MRCP diagnosed all 22 cases. The Sensitivity and specificity for Ultrasonography was $71 \%$ and $100 \%$ respectively in detection of biliary obstruction secondary to biliary strictures and for MRCP, the sensitivity and specificity was $100 \%$. [Figure 2]. However in differentiation of benign vs malignant strictures, MR under diagnosed a periampullary carcinoma as benign biliary stricture.

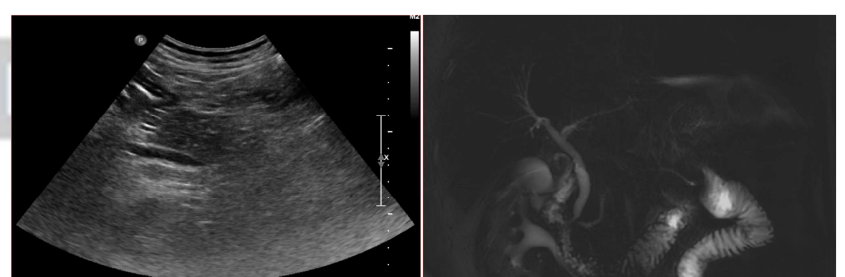

Figure 2: USG image showing dilated CBD with smooth tapering of Distal CBD- s/o Benign stricture. 3D-SSH MRCP image showing smooth narrowing of distal CBD- s/o Benign stricture.
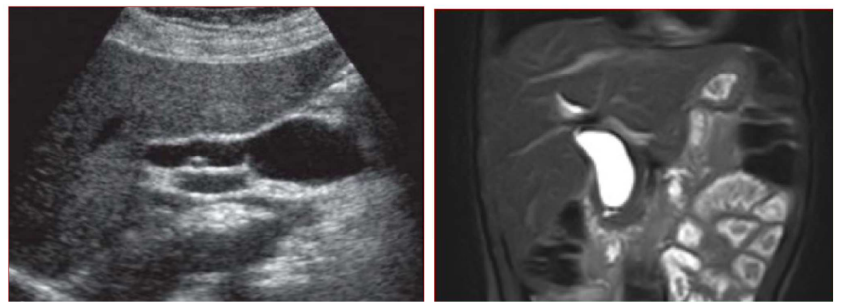

Figure 3: USG imgae showing fusiform dilatation of CBD- s/o Type I Choledochal cyst. T2WI- Fat saturated coronal section shows fusinform dilatation of CBD- s/o TypeI Choledochal Cyst.

Two patients with Choledochal cysts were accurately diagnosed by both Ultrasonography and MRCP.

3 patients were diagnosed to have Mirizzi's on 


\section{Patil et al; Rale of Ultrasanagraphy in the Era of 3 T esla MRPS}

Ultrasonography, of these only 2 cases were true positive. MRCP diagnosed both the cases, the sensitivity and specificity in this study was $66 \%$ \& 100 (Ultrasonography), $100 \%$ for MRCP. One patient diagnosed to have Mirizzi's (False positive) was only choledocholithiasis on MRCP. Correlative accuracy rates could not be commented on owing to a small sample (only 2 cases).

Out of 5 biopsy proven cases of periampullary carcinoma Ultrasonography was able to identify the lesion in only 3 cases where as MRCP was able to identify 4 out of 5 cases giving a diagnostic accuracy of $60 \%$ for Ultrasonography and $80 \%$ for MRCP. One case was reported as benign stricture in MRCP, owing to the smooth tapering of distal CBD and a normal pancreatic duct, however ERCP periampullary growth was noted.
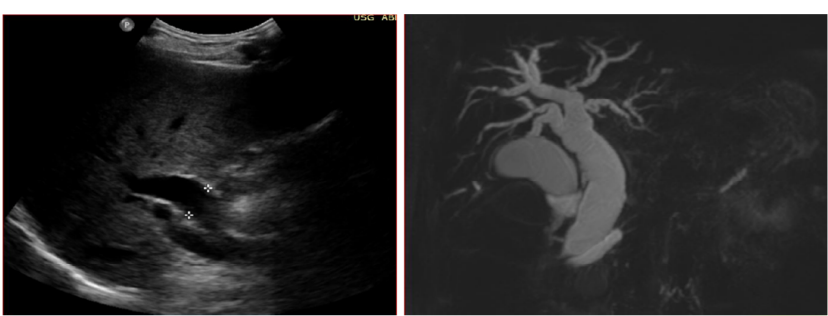

Figure 4: USG image shows dilated Proximal and mid CBD with suboptimal Distal CBD, while 3D- SSH image in coronal section shows abrupt cut-off of distal CBD, Dilated Main pancreatic duct and IHBRD.

Of the 5 cases of histopathologically proven Cholangiocarcinoma, 3 were diagnosed on Ultrasonography and MRCP diagnosed all 5 cases. Two cases of intrahepatic cholangiocarcinoma were diagnosed as HCC on ultrasound, as the lesions were vascular and hyperechoic lacking the classical features of cholangiocarcinoma like heterogeneous lesion, minimal internal vascularity, lobar atrophy and capsular retraction were not there. Sonographic accuracy of intrahepatic cholangiocarcinoma, sclerosing distal cholangiocarcinoma or periampullary carcinoma is limited.
3 cases of biopsy Histopathology proven Carcinoma gall bladder were correctly detected in both Ultrasonography as well MRCP. Of the 2 cases of carcinoma head of the pancreas, Both Ultrasonography and MRCP could accurately diagnose both the cases of carcinoma head of the pancreas.

Ultrasonography as a screening modality is useful to confirm or exclude biliary dilatation and to choose patients for MRCP examination. MRCP is an important noninvasive imaging investigation in the pre-operative evaluation of patients with obstructive jaundice.

The sensitivity, specificity and diagnostic accuracy for choledocholithiasis is $79 \%, 100 \%$ \& $80 \%$ respectively for Ultrasonography and $100 \%$ for MRCP. Sensitivity, specificity and diagnostic accuracy was more for MRCP in comparison to sonography owing to reasons enlisted above. Sensitivity and specificity for benign biliary strictures in this study was $71 \%$ and $100 \%$ respectively on Ultrasonography as opposed to MRCP which was $100 \%$. MRCP is more sensitive in detection of CBD strictures than Ultrasonography, while specificity rates for Ultrasonography and MRCP remains same.

Results in the study showed a overall sensitivity was $79 \%$, specificity was $100 \%$ and accuracy was $78 \%$ for cases with benign conditions on ultrasound. While, sensitivity was $93.3 \%$, specificity was $100 \%$ and accuracy was $93.3 \%$ for cases with benign conditions on MRCP. Of the 16 cases with benign aetiology missed on ultrasound, 10 were choledocholithiasis and 6 were benign biliary strictures. The major limitation encountered was poor acoustic window secondary to bowel gas and body habitus (obese patients), five of these patients who recalled for repeat scan were scheduled for MRCP, the patients with strictures a differential of ductal stone / sludge was inferred on ultrasound, the terminal duct could not be imaged owing to bowel gas. Compression ultrasound was also impeded owing to higher BMI of patients evaluated.

Table 3: Comparison of Diagnostic values of USG VS MRCP- Benign causes

\begin{tabular}{|c|c|c|c|c|c|c|c|}
\hline Modality & $\begin{array}{c}\text { Sensitivity } \\
(\%)\end{array}$ & $\begin{array}{c}\text { Specificity } \\
(\%)\end{array}$ & $\begin{array}{l}\text { Positive predictive } \\
\text { value (PPV) (\%) }\end{array}$ & $\begin{array}{l}\text { Negative predictive } \\
\text { value (NPV) }(\%)\end{array}$ & $\begin{array}{c}\text { Accuracy } \\
(\%)\end{array}$ & $\begin{array}{c}\text { False positive cases } \\
\text { No. }\end{array}$ & $\begin{array}{c}\text { False negative } \\
\text { cases No. }\end{array}$ \\
\hline USG & 79 & 100 & 97 & 75.6 & 78 & 1 & 16 \\
\hline MRCP & 100 & 100 & 100 & 100 & 100 & 1 & 0 \\
\hline
\end{tabular}

Table 4: Comparison of Diagnostic values of USG VS MRCP- Malignant causes

\begin{tabular}{|c|c|c|c|c|c|c|c|}
\hline Modality & $\begin{array}{c}\text { Sensitivity } \\
(\mathbf{\%})\end{array}$ & $\begin{array}{c}\text { Specificity } \\
(\%)\end{array}$ & $\begin{array}{c}\text { Positive predictive } \\
\text { value (PPV) (\%) }\end{array}$ & $\begin{array}{c}\text { Negative predictive } \\
\text { value (NPV) (\%) }\end{array}$ & $\begin{array}{c}\text { Accuracy } \\
(\mathbf{\%})\end{array}$ & $\begin{array}{c}\text { False positive } \\
\text { cases No. }\end{array}$ & False negative cases No. \\
\hline USG & 73 & 100 & 100 & 80.5 & 73.3 & 0 & 4 \\
\hline MRCP & 93.3 & 100 & 100 & 100 & 93.3 & 0 & 1 \\
\hline
\end{tabular}

Sensitivity and specificity for malignant pathologies in this study was $73 \%$ and $100 \%$ respectively on Ultrasonography while that on MRCP was $93.3 \%$ and $100 \%$ respectively. From this it is clear that MRCP is more sensitive in detection of pancreatico-biliary malignancy while specificity of both Ultrasonography and MRCP remains same. Verma et $a{ }^{[30]}$ demonstrated the sensitivity and specificity of $88.4 \%$ and $85.3 \%$ on ultrasound, $86 \%$ and $92 \%$ on MRCP respectively for detecting the malignant etiology of obstruction. [Table 7]

MRCP is a rapidly developing non-invasive modality for evaluation of pancreatico-biliary diseases. Biliary obstruction represents one of the main indications for MRCP. It is able to produce highly accurate 
cholangiographic images, similar to that at direct cholangiography and yet combine the patient comfort and safety associated with ultrasound. Complete mapping of the ductal system, non-invasiveness, no requirement of intravenous contrast and lack of exposure to ionizing radiation are some of its important features. Its inability to offer therapeutic intervention is its only drawback when compared to ERCP.

Magnetic resonance (MR) cholangiopancreatography, which combines the advantages of projectional imaging with those of cross-sectional imaging, is an established diagnostic technique that can be substituted for endoscopic retrograde cholangiopancreatography (ERCP) in most clinical settings. Technical advances over the past decade, including the refinement of 3.0-T imaging capabilities, have added to the usefulness of this noninvasive method. MR cholangiopancreatography is now considered the imaging test of choice after a failed or incomplete ERCP evaluation. It is also helpful for pre surgical planning and postsurgical follow-up.

Limitations of USG are due to non visualization of the most distal part of CBD (due to overlying bowel gas or mass lesion) while MRCP define the whole duct, USG is operator dependent and Calculus in non-dilated bile duct may be missed by USG.

Limitations of MRI: Claustrophobia, breath holding is difficult in children, elderly and debilitated patients, Costly, time consuming and has MRI related artefacts

\section{Conclusion}

Ultrasound still remains the primary investigative modality of choice with acceptable levels of diagnostic accuracy. Limitation of ultrasound would be in obese patients/ bowel gas.

3 Tesla MRI/MRCP is superior to ultrasound in studying both the benign and malignant lesions. The limitations are high cost, non availability.

Though MRCP can be considered as the gold standard for diagnostic non-invasive evaluation of the pancreaticobiliary system in cases of obstructive jaundice, ultrasound will always remain the first choice.

\section{References}

1. Watanable $\mathrm{Y}$ et al. MR imaging of acute biliary disorders. RadioGraphics. 2007; 27: 477-95.

2. Upadhyay $\mathrm{V}$ et al. Comparative Assessment of imaging modalities in biliary obstruction. Indian J Radiol Imaging. 2006:16(4):577-82.

3. Bhatt et al. Comparison of Diagnostic accuracy between Ultrasonography and MRCP in biliary and pancreatic pathology. Indian J Radiol Imaging. 2005; 15 (2):178-81.

4. Sutton D, Textbook of Radiology and Imaging, 7th edition, 2003 and
United Kingdom, Elsevier sciences ltd.

5. Yi Tang, MD et al. Pancreaticobiliary Ductal System: Value of HalfFourier Rapid Acquisition with Relaxation Enhancement MR Cholangiopancreatography for Postoperative Evaluation. RadioGraphics. 2000;215(1): 81-88.

6. J A Larena, E Astigarraga, I Saralegui, A Merino, A Capelastegui, and M M Calvo. Magnetic resonance cholangiopancreatography in the evaluation of pancreatic duct pathology. Br J Radiol. 1998; 71(850): 1100-04.

7. Ahmet Mesrur Halefoglu. Magnetic resonance cholangiopancreatography: A useful tool in the evaluation of pancreatic and biliary disorders. World $\mathrm{J}$ Gastroenterol. 2007;13(18):2529-34.

8. Kouji Morimoto, MD et al. Billary Obstruction: Evaluation with Three-dimensional MR Cholangiography. RadioGraphics. 1992:183:578-80.

9. Takhera $\mathrm{Y}$ et al. Breathhold MRCP with a long echo train fast spin echo sequences and surface coil in chronic pancreatitis. Radiology. 1994; 192:73-78.

10. Morimoto et al. Single Shot RARE VS Multislice HASTE in evaluation of Choledocholithiasis. Clinical Radiology. 2000;55:86673.

11. Patil V, Shabadi N, Das S, Shetty S, Sen K. Magnetic Resonance Cholangiopancreatography in 3 Tesla: 2D MRCP versus 3D MRCP in Diagnostic Evaluation with Special Reference to Different Acquisition and Reconstruction Planes. J Glob Radiol. 2015;1(1):Article 3

12. Fulcher AS, Turner MA. MR pancreatiography a useful tool for evaluating pancreatic disorders. Radiographics. 1999; 19(1):5-24.

13. A. Arslan, J.T. Geitung, E. Viktil, M. Abdelnoor \& M. Osnes. Pancreaticobiliary Diseases: Comparison of 2D single-shot turbo spinecho MR cholangiopancreatography with endoscopic retrograde cholangiopancreatography. Acta Radiologica. 2000; 41(6):621-626.

14. Gilloteaux J. Introduction to the biliary tract, the gallbladder, and gallstones. Microsc Res Tech. 1997; 38: 547-51.

15. Saragawi Radha et al. Anatomical Variations of Cystic Ducts in Magnetic Resonance Cholangiopancreatography and Clinical Implications. Radiology Research and Practice. 2016; 3021484(id): 16.

16. Berry Manorama, Suri Sudha, Chowdhary Veen. Diagnostic Radiology: Gastrointenstinal and Hepatobiliary Imaging.New Delhi: Jaypee Brothers Medical Publishers(P) Ltd; 2009.

7. Keith Quencer, MD et al. Imaging of the pancreas: Part 1. Applied Radiology.2013;42(9):14-20.

18. Manfredi R et al. Pancreas divisum and "Santorinicele": diagnosis with dynamic MR cholangiopancreatography with secretin stimulation. Radiology. 2000; 217(2):403-8.

19. Simpson D, Radiology of the pancreas, Gall bladder and biliary tract. Radiol Clin N Am. 2002;40:6

20. Rumack C, CharboneauW, Wilson S. Diagnostic Ultrasound Volume 1,3rd edition.2005; page 240

21. Bachar GN, Cohen M, Belenky A, Atar E, Gideon S. Effect of aging on the adult extrahepatic bile duct: a sonographic study. J Ultrasound Med. 2003; 22(9):879-82.

22. Onofrio A. Catalano, MD et al. MR Imaging of the Gallbladder: A Pictorial Essay. RadioGraphics. 2008; 28(1): 135-56.

23. Jon W. Meilstrup, Kenneth D. Hopper, and Gary A. Thieme. Imaging of Gallbladder Variants. Am J Roentgenol. 1991; 157:1205-08.

24. Abboud PA, Malet PF, Berlin JA, Staroscik R, Cabana MD, Clarke JR, Shea JA,Schwartz JS, Williams SV. Predictors of common bile duct stones prior tocholecystectomy: a meta-analysis. Gastrointest Endosc. 1996;44(4):450-5

Copyright: (C) the author(s), publisher. Asian Journal of Medical Radiological Research is an Official Publication of "Society for Health Care \& Research Development". It is an open-access article distributed under the terms of the Creative Commons Attribution Non-Commercial License, which permits unrestricted non-commercial use, distribution, and reproduction in any medium, provided the original work is properly cited.

How to cite this article: Patil V, Das SK, Devkant, Hiremath R, Shetty S, Raj V. Role of Ultrasonography in the Era of 3 Tesla MRCP: A Comparative Study in Obstructive Jaundice. Asian J. Med. Radiol. Res. 2019;7(1):09-15.

DOI: dx.doi.org/10.21276/ajmrr.2019.7.1.3

Source of Support: Nil, Conflict of Interest: None declared. 